\title{
Molecular Screening of Nasopharyngeal Carcinoma: Detection of LMP-1, LMP-2 Gene Expression in Vietnamese Nasopharyngeal Swab Samples
}

\author{
Thuan Duc Lao ${ }^{1}$, Tuan Anh Hoang Nguyen², Kha Dong Ngo², Hue Hong Thieu², \\ Minh Trong Nguyen ${ }^{3}$, Dung Huu Nguyen ${ }^{3}$, Thuy Ai Huyen Le ${ }^{1 *}$
}

\begin{abstract}
Objective: This study aimed to characterize the expression of $L M P-1, L M P-2$ in clinical swab samples in order to find out the potential molecular based biomarker for NPC diagnosis and screening, which could offer a chance in development of rapid method for NPC diagnosis in Vietnamese population. Materials and Methods: A total of 93 nasopharyngeal carcinoma swab samples and 100 healthy nasopharyngeal swab samples were collected to evaluate $L M P-1, L M P-2$ expression by Real-time reversed PCR. Results: we figured out the significant association between the expression of $L M P-1$ (counting for $48.39 \%$ ), LMP-2 (counting for $39.78 \%$ ) and NPC. No $L M P-1$ expression was observed, and only 1 of 100 specimens was detected with LMP-2 positive in healthy samples. In the combination of $L M P-1(+)$ and/or LMP-2 (+), the frequency of positive was $53.76 \%$, greater than each gene expression. Additionally, sensitivity, specificity, positive predictive value, negative predictive value of assay were $99.00 \%, 98.04 \%, 69.72 \%$, and $77.02 \%$, respectively. Additionally, the $L M P-2$ expression level was 5.50 times higher in NPC samples than non-cancerous samples. Conclusion: Our results indicated the molecular invasive method based on the expression of $L M P-1, L M P-2$ in swab samples would be a promising supplement in NPC diagnosis, screening in the near future in Vietnam.
\end{abstract}

Keywords: $L M P-1-L M P-2$ - Epstein-Barr virus- nasopharyngeal swab sample- Vietnam

Asian Pac J Cancer Prev, 20 (9), 2757-2761

\section{Introduction}

A well-established etiology factor of NPC is its strong associated to Epstein-Barr virus (EBV), also known as human gamma herpes virus 4 (HHV4), has been postulated since the year of 1966 (Rowe et al., 1992; Vera-Sempere et al., 1996; See et al., 2008, Young, Dawson, 2014; Mahdavifar et al., 2016). EBV remains two alternative lifestyles: (1) the latency stage; (2) the lytic stage or productive phrase of EBV (Tsurumi et al., 2005). Between two stages, EBV establishes life-long latent persistence (Bocian, Januszkiewicz-Lewandowska, 2011). In latency state, EBV genes are expressed in the limited set of viral latent transcripts (Young et al., 2000; Raab-Traub, 2002; Marquitz and Raab-Traub, 2012). Among the latent genes products, LMP-1 (Latent membrane protein-1) and LMP-2 (Latent membrane protein-2) are consistently expressed in subset of NPC tumors, therefore, they display useful oncogenic targets of NPC diagnosis (Brooks et al., 1992; Hao et al., 2004; See et al., 2008; Lao et al., 2017).
$L M P-1$ encoded its latent membrane protein that induce morphological and phenotypic alterations in epithelial cells (Hao et al., 2004; Kang, Kieff, 2015; Dawson et al., 2012). The function of LMP-2 is considered as playing important role in carcinogenesis by driving EBV into latency (Thompson, Kurzrock, 2004; Dawson et al., 2012). Many previous studies showed that LMP-1, LMP-2 has been considered as the molecular prognostic, diagnostic as well as the outcome of therapy in NPC (Hao et al., 2004; Hariwiyanto et al., 2010; Rosales-Pérez et al., 2014; Lao et al., 2017).

Vietnam, located in Southern Asia, is well known as the high incidence and mortality rate of nasopharyngeal carcinoma within 86,691 cases (Age-standardized rate $-\mathrm{ASR}=1.2 / 100,000)$ and $50,831(\mathrm{ASR}=0.7 / 100,000)$ deaths (Mahdavifar et al., 2016; Globocan, 2012). In our previous, we reported our experience of detecting EBV-derived genomic DNA, includes EBNA-1, EBNA-2, $L M P-1$ and $L M P-2$, counting for $46.32 \%, 49.47 \%, 45.26 \%$ and $47.37 \%$, respectively, by nasopharyngeal swab in NPC

${ }^{1}$ Department of Pharmaceutical and Medical Biotechnology, Faculty of Biotechnology, Ho Chi Minh City Open University, Ho Chi Minh City, ${ }^{2}$ Faculty of Biology and Biotechnology, University of Science, VNU-HCM, ${ }^{3}$ Cho Ray Hospital, HCMC, Vietnam. *For Correspondence: thuy.lha@ou.edu.vn 
patients (Lao et al., 2017). Moreover, low frequencies of those genes were observed in non-cancerous samples. We also proved that the EBNA-1, EBNA-2, $L M P-1$, $L M P-2$ based detection are useful oncogenic targets to screen NPC (Lao et al., 2017). EBV infection in NPC has been classified as the latency type 2 infection in which only EBNA-1, LMP1, LMP2 expressions could be detected (Korcum et al., 2006). It may throw light on the development of mRNA EBV expression based molecular screening, diagnosis, prognosis for NPC in the high-incidence areas, includes Vietnam. Continuing with our previous study, for the aims to find out the potential molecular biomarkers for NPC management, we defined the EBV latency-derived $L M P-1, L M P-2$ gene expression whether or not associated with NPC in Vietnamese NPC patients. Especially, we carried out on nasopharyngeal swab samples, the non-invasive samples, and it would be a good supplement and promising role in the further NPC screening and diagnosis.

\section{Materials and Methods}

\section{Ethics statement, samples collection}

Institutional Ethics Board approval was obtained from the Medical Ethics Committee of the Cho Ray Hospital, Ho Chi Minh City, Vietnam. (The decision number of the permission from Ethical committee: 516/BVCR-HDDD, Cho Ray Hospital, Ho Chi Minh City, Vietnam). All the samples used in this study were agreed by Cho Ray Hospital and obtained from all participants in current study. The patient, who was enrolled in this study, are required to be agreed and sign on the consent forms to approve the usage of the samples for laboratory work and analysis.

A total of 93 NPC swab samples, confirmed by immunohistochemistry, were archived and admitted from the Cho Ray Hospital, Vietnam. For 100 non-malignant swab samples, which were negative for nasopharyngeal carcinoma, were collected from non-NPC patients. In brief, a 15-cm-long cotton stick was inserted into the nasal cavity and moved toward the nasopharyngeal wall, then, swept over the surface of the posterior and lateral nasopharyngeal wall. The cotton stick was withdraw and immediately immersed in the phosphate-buffered saline solution, stored at $-20^{\circ} \mathrm{C}$ for further experiments.

Total RNA extraction, real-time PCR assay

Total of RNA was extracted by using TRIzolTM Reagent (Cat: 15596026, Invitrogen). cDNA was reverse transcribed from approximately $5 \mathrm{ng}$ of Total RNA by using The High Capacity cDNA Reverse Transcription Kit (Thermo Fisher Scientific). All the RNA extraction and the reverse transcriptions assays were performed according to the manufacturer's instructions. For cDNA $L M P-1, L M P$-2 detection, qRT-PCR reactions were done by means of a qSYBR-green, and GAPDH was used as an endogenous control. The internal control candidate was used to normalize the Ct values of each $L M P-1, L M P-2$. The primers, which were used in current study, were obtained by previous study (Table 1).

\section{Statistical analysis}

Data were analyzed using Medcalc ${ }^{\circledR}$ Version 12.7.0.0. All $\mathrm{p}$ values were two-side, and less than 0.05 were considered statistically significant. All values were reported as mean $\pm \mathrm{SEM}$. The relative expression of $L M P-1, L M P-2$ as determined using q-PCR was analyzed using the $2^{-\Delta \Delta \mathrm{Ct}}$ method. Finding was greater and less than 1 was determined to classify up-regulation and down-regulation, respectively. Chi-test was used to determine the association between the expression of $L M P-1, L M P-2$ and NPC status. Moreover, the association between expression of $L M P-1, L M P-2$ and risk of NPC was estimated by computing OR, RR and $95 \%$ confidence intervals $(\mathrm{CI})$.

\section{Results}

\section{Patient characteristics}

The characteristics of the total of 93 NPC patients were summarized in Table 2. The mean age of 93 NPC case was $53.51 \pm 1.43$ (range: from 20 to 81). Among them, the number of male (counting for $73.12 \%$ ) is more than female (counting for $26.88 \%$ ) by 2.72 times. The age incidence profile indicated the increase of NPC risk by the age up to the late middle age (range: from 40 to under 60). Tumor histological types were classified according to World Health Organization (WHO) classification for NPC criteria. Type 3 (undifferentiated carcinoma: UC) counted for the highest proportion of all types of NPC. $67.74 \%(n=$ 63) NPC cases were classified as type 3 (undifferentiated carcinoma), occupied the highest proportion of its cases. Stage wise, $48.39 \%(n=45)$ NPC patients was observed in an advanced stage, and no case was observed in the early stage (stage 1).

Epstein-Barr virus genes: LMP-1, LMP-2 expression were highly expressed in NPC clinical swab samples

The frequencies of $L M P-1, L M P-2$ expression were shown in Table 3. In the NPC swab set, 45 of 93 NPC specimens (counting for $48.39 \%$ ) were positive for LMP-1 expression, whereas none of the controls was positive. 37 of 93 NPC samples (counting for 39.78\%), 1 of 100 non-cancerous samples (counting for $1.00 \%$ ) were positive for LMP-2 expression, respectively. Table 3 showed the validity of expression each gene in NPC clinical swab samples. A value of $p<0.0001$ indicated that the each gene expression were significantly associated with NPC. Using the $L M P-1$ gene and later rectification with $L M P-2$ expression [LMP-1 (+) and/or LMP-2 (+): RPI (Real-time PCR Index) $\geq 0.5$, meant that at least one of two genes were expressed], NPC could be diagnosed with a sensitivity of $53.76 \%$ (50/93), specificity of $99.00 \%$ (99/100), positive predictive value of 98.04\% (50/51), negative predictive value of $69.72 \%(99 / 142)$, and accuracy of $77.02 \%$ (149/193). No significant association was found between $L M P-1, L M P-2$ expression and other clinical characteristics, such as patient's gender, age, tumor histological types, as well as stage $(\mathrm{p}>0.05)$.

No significant association was found between $L M P-1$, $L M P-2$ expression and other clinical characteristics, such as patient's gender (LMP-1: $p=0.06$; LMP-2: $p=0.83$ ), 
Table 1. The Sequences of Primers Used in Current Study

\begin{tabular}{ll}
\hline Primer & Sequences $\left(5^{\prime}-3^{\prime}\right)$ \\
\hline LMP-1-F & CAGTCAGGCAAGCCTATGA \\
LMP-1-R & CTGGTTCCGGTGGAGATGA \\
LMP-2-F & AGCTGTAACTGTGGTTTCCATGAC \\
LMP-2-R & GCCCCCTGGCGAAGAG \\
\hline
\end{tabular}

F, Forward primer; R, Reverse primer

Table 2. Characteristics of NPC Patients

\begin{tabular}{llc}
\hline Variables & & $\mathrm{n}(\%)$ \\
\hline Sex & Male & $68(73.12)$ \\
& Female & $25(26.88)$ \\
Age & $\leq 20$ & $1(1.08)$ \\
& 20 to $\leq 40$ & $17(18.28)$ \\
& 40 to $\leq 60$ & $43(46.23)$ \\
& 60 to $\leq 80$ & $31(33.33)$ \\
Pathological classification & $>80$ & $1(1.08)$ \\
& Type 1 & $4(4.30)$ \\
& Type 2 & $26(27.96)$ \\
Stage & Type 3 & $63(67.74)$ \\
& I & $0(0.00)$ \\
& II & $33(35.48)$ \\
& III & $15(16.13)$ \\
& IV & $45(48.39)$ \\
\hline
\end{tabular}

Type 1, keratinizing squamous cell carcinoma; Type 2, non- keratinizing carcinoma; Type 3, undifferentiated carcinoma

age $(L M P-1: \mathrm{p}=0.19 ; L M P-2: \mathrm{p}=0.26)$, tumor histological types $(L M P-1: \mathrm{p}=0.29 ; L M P-2: \mathrm{p}=0.28)$, as well as stage $(L M P-1: \mathrm{p}=0.07 ; L M P-2: \mathrm{p}=0.40)$.

The mean $\mathrm{Ct}$ values for GAPDH in the case and control group were $27.14 \pm 2.31$ and $27.75 \pm 2.24$, respectively. No significant difference in the GAPDH expression was found between case and control group $(\mathrm{p}=0.77>0.05)$. Therefore, GAPDH was suitable as a reference gene (internal control) to normalize the $L M P-1$ and $L M P-2$ expression between the case and control groups. The meant $\mathrm{Ct}$ value of $L M P-1$ in the case was 25.14, and no $\mathrm{Ct}$ value was recorded due to no control samples were positive for $L M P-1$ expression. Although LMP-1 was not detected in control group, it also could be confirmed that the expression of $L M P-1$ was elevated in patient samples. The relative expression of $L M P-2$ was computed by the $2^{-\Delta \Delta \mathrm{Ct}}$ method based on the comparison of
Expression of LMP-1, LMP-2 in Nasopharyngeal Swab Samples

$L M P-2$ expression in case and control group. The mean of $\mathrm{Ct}$ value of $L M P$-2 in the case and control group were 24.93 and 27.97, respectively. As the result, the $L M P-2$ expression level was 5.50 times higher in NPC samples than non-cancerous samples $\left(2^{-\Delta \Delta \mathrm{Ct}}=5.50, \mathrm{p}<0.05\right)$.

\section{Discussion}

Up to date, there are challenging to diagnosis NPC, because of the vague symptoms such as hearing loss, bloody nasal discharge, diplopia and headache. Especially, in Vietnam, the high incidence and mortality rate of NPC, most patients present at an advance stage when first diagnosis (Stage 3, or stage 4). There is essential to find an early diagnosis and biomarker to achieve favorable treatment and increasing of patient's survival. Many previous studies have been reported that the association between NPC and EBV may throw light on the molecular diagnosis of NPC (Hildesheim and Levine, 1993; Hao et al., 2004; Lao et al., 2017; Wu et al., 2018). According to the function of $L M P-1$, its encoded protein plays a key role as a viral mimic of the TNFR family member, CD40, engaging a number of signaling pathways, such as NF-kB, JNK, p38 pathway (Hao et al., 2004; Xu et al., 2006; Dawson et al., 2012; Kang and Kieff, 2015). LMP2 plays important role in carcinogenesis by driving EBV into latency and provides essential survival signals through the constitutive activation of the ERK/MAPK pathway (Thompson and Kurzrock, 2004; Dawson et al., 2012). In the latent stage, $L M P-1, L M P-2$ expression, which are expressed in EBV latency type II, exemplified by NPC, contribute to cell survival (Kang and Kieff, 2015). Thus, $L M P-1$ and $L M P-2$ are thought to be the meaningful biomarker for NPC management.

The use of NPC biopsy samples were considered as the "standard" sample in NPC diagnosis, based on imaging diagnosis, immunohistochemistry, etc. However, the non-invasive samples, such as peripheral blood, nasopharyngeal swabs, throat swabs, plasma and saliva have been used for detecting the etiological causes of NPC, including the presence of EBV (Liu et al., 2013; Zheng et al., 2015; Lao et al., 2017). Therefore, in current study, the nasopharyngeal swab samples were used in the detection of $E B V L M P-1, L M P-2$ expression to find out the non-invasive, potential biomarker for NPC diagnosis and screening.

In current study, this is the first case - control study was carried out to evaluate whether or not $L M P-1$, $L M P-2$ expression could be further applied in NPC

Table 3. Data Analysis of $L M P-1, L M P-2$

\begin{tabular}{lcccccc}
\hline & \multicolumn{2}{c}{$L M P-1$} & \multicolumn{2}{c}{$L M P-2$} & \multicolumn{2}{c}{$\mathrm{RPI} \geq 0.5$} \\
& $\mathrm{P}(\%)$ & $\mathrm{N}(\%)$ & $\mathrm{P}(\%)$ & $\mathrm{N}(\%)$ & $\mathrm{P}(\%)$ & $\mathrm{N}(\%)$ \\
\hline NPC & 45 & 48 & 37 & 56 & 50 & 43 \\
$(\mathrm{n}=93)$ & $(48.39)$ & $(51.61)$ & $(39.78)$ & $(60.22)$ & $(53.76)$ & $(46.24)$ \\
Control & 0 & 100 & 1 & 99 & 1 & 99 \\
$(\mathrm{n}=100)$ & $(0.00)$ & $(100.00)$ & $(1.00)$ & $(99.00)$ & $(1.00)$ & $(99.00)$ \\
$\mathrm{p}$ & $<0.0001$ & & $<0.0001$ & & $<0.0001$ & \\
\hline
\end{tabular}


screening, diagnosis as well as therapy in Vietnam. As the result, we pointed out the significant association between the expression of $L M P-1$ (counting for $48.39 \%$ ), LMP-2 (counting for $39.78 \%$ ) and NPC. Whereas, in the case of control group, no LMP-1 expression was observed, and only 1 of 100 specimens was detected with $L M P-2$ positive $(\mathrm{p}<0.0001)$. Additionally, the LMP-2 expression was confirmed to be 9.78 times higher in tumor samples, compared to non-cancerous samples $\left(2^{-\Delta \Delta \mathrm{Ct}}=5.50\right.$, $\mathrm{p}<0.05)$. Although $L M P-1$ was not detected in control group, it also could be confirmed that the expression of $L M P-1$ was elevated in patient samples. In the combination of LMP-1 (+) and/or LMP-2 (+) (RPI (Real-time PCR Index) $\geq 0.5)$, the frequency of positive was $53.76 \%$, greater than each gene expression. Additionally, sensitivity, specificity, positive predictive value, negative predictive value of assay were $99.00 \%, 98.04 \%, 69.72 \%$, and $77.02 \%$, respectively. Thus, it indicated that the oncogenic role of $L M P-1, L M P-2$ in NPC was well evaluated and confirmed in this case - control group study. Therefore, our investigations reveal that LMP-1, $L M P$-2 expression-based invasive method was identified as further promising biomarker for prognosis, diagnosis and therapy for NPC.

In conclusion, in current study, we found out the significant association between $L M P-1, L M P-2$ expression as well as $L M P-1$ and/or $L M P-2$ expression and NPC in Vietnamese NPC patients. Our results pointed out the significant association between $L M P-1$, and $L M P-2$ expression and NPC, performed by Real-time reverse PCR method. This molecular-based invasive method would be a promising supplement in NPC diagnosis, screening in the near future in Vietnam.

\section{Acknowledgements}

We wish to express our thanks to the research project sponsored by Ho Chi Minh City Open University. We thank all the recruited participants in this work and Dr. Nguyen Trong Minh, Dr. Nguyen Huu Dung, and all the staff members of Otorhinolaryngology in Cho Ray hospital, Ho Chi Minh City, for collecting the samples used in these studies. We are thankful to the participants in our study and appreciated the contributions of the staffs from Molecular Biology Laboratory, Ho Chi Minh City Open University.

\section{Ethics approval and consent to participate}

All patients signed inform consent before entering into the study. No study drug or procedure was applied. This is an observational study.

\section{Acknowledgments}

Conflict of interest

The authors declared that they have no competing interests.

\section{References}

Bocian J, Januszkiewicz-Lewandowska D (2011). Epstein-Barr virus infection - life cycle, methods of diagnosis, associated diseases. Postepy Hig Med Dosw, 65, 286-98.

Brooks L, Yao QY, Rickinson AB, Young LS (1992). Epstein-Barr virus latent gene transcription in nasopharyngeal carcinoma cells: coexpression of EBNA1, LMP1, and LMP2 transcripts. J Virol, 66, 2689-97.

Dawson CW, Port RJ, Young LS (2012). The role of the EBV-encoded latent membrane proteins LMP1 and LMP2 in the pathogenesis of nasopharyngeal carcinoma (NPC). Semin Cancer Biol, 22, 144-53.

GLOBOCAN (2012). Estimated cancer incidence, mortality and prevalence worldwide in 2012, http://globocan.iarc.fr/ Pages/fact_sheets_population.aspx.

Hao SP, Tsang NM, Chang KP, et al (2004). Molecular diagnosis of nasopharyngeal carcinoma: detecting $L M P-1$ and $E B N A$ by nasopharyngeal swab. Otolaryngol Head Neck Surg, 131, 651-4.

Hariwiyanto B, Sastrowiyoto S, Mubarika S, et al (2010). LMP1 and LMP2 may be prognostic factors for outcome of therapy in nasopharyngeal cancers in Indonesia. Asian Pac J Cancer Prev, 11, 763-6.

Hildesheim A, Levine PH (1993). Etiology of nasopharyngeal carcinoma: a review. Epidemiol Rev, 15, 466-85.

Kang MS, Kieff E (2015). Epstein-Barr virus latent genes. Exp Mol Med, 47, e131.

Korcum AF, Özyar E, Ayhan A (2006). Epstein-Barr virus genes and nasopharyngeal cancer. Turk J Cancer, 36, 97-107.

Lao TD, Nguyen DH, Nguyen TM, et al (2017). Molecular Screening for Epstein-Barr virus (EBV): Detection of Genomic EBNA-1, EBNA-2, LMP-1, LMP-2 Among Vietnamese Patients with Nasopharyngeal Brush Samples. Asian Pac J Cancer Prev, 18, 1675-9.

Liu X, Luo HN, Tian WD, et al (2013). Diagnostic and prognostic value of plasma microRNA deregulation in nasopharyngeal carcinoma. Cancer Biol Ther, 14, 1133-42.

Mahdavifar N, Ghoncheh M, Mohammadian-Hafshejani A, et al (2016). Epidemiology and inequality in the incidence and mortality of nasopharynx cancer in Asia. Osong Public Health Res Perspect, 7, 360-72.

Marquitz AR, Raab-Traub N (2012). The role of miRNAs and EBV BARTs in NPC. Semin Cancer Biol, 22, 166-72.

Raab-Traub, N (2002). Epstein-Barr virus in the pathogenesis of NPC. Semin Cancer Biol, 12, 431-41.

Rosales-Pérez S, Cano-Valdez AM, Flores-Balcázar CH, et al (2014). Expression of Epstein-Barr virus-encoded latent membrane protein (LMP-1), p16 and p53 proteins in nonendemic nasopharyngeal carcinoma (NPC): a clinicopathological study. Arch Med Res, 45, 229-36.

Rowe M, Lear AL, Croom-Carter D, et al (1992). Three pathways of Epstein-Barr virus gene activation from EBNA1-positive latency in B lymphocytes. J Virol, 66, 122-31.

See HS, Yap YY, Yip WK, et al (2008). Epstein-Barr virus latent membrane protein-1 (LMP-1) 30-bp deletion and Xho I-loss is associated with type III nasopharyngeal carcinoma in Malaysia. World J Surg Oncol, 6, 18.

Thompson MP, Kurzrock R (2004). Epstein-Barr virus and cancer. Clin Cancer Res, 10, 803-21.

Tsurumi T, Fujita M, Kudoh A (2005). Latent and lytic EpsteinBarr virus replication strategies. Rev Med Virol, 15, 3-15.

Vera-Sempere FJ, Burgos JS, Botella MS, et al (1996). Immunohistochemical expression of Epstein-Barr virusencoded latent membrane protein (LMP-1) in paraffin sections of EBV-associated nasopharyngeal carcinoma in Spanish patients. Eur J Cancer B Oral Oncol, 32, 163-8.

Wu L, Li C, Pan L (2018). Nasopharyngeal carcinoma: A review of current updates. Exp Ther Med, 15, 3687-92.

Xu D, Brumm K, Zhang L (2006). The latent membrane protein 
1 of Epstein-Barr virus (EBV) primes EBV latency cells for type I interferon production. J Biol Chem, 281, 9163-9.

Young LS, Dawson CW (2014). Epstein-Barr virus and nasopharyngeal carcinoma. Chin J Cancer, 33, 581-90.

Young LS, Dawson CW, Eliopoulos AG (2000). The expression and function of Epstein-Barr virus encoded latent genes. $\mathrm{Mol}$ Pathol, 53, 238-47.

Zheng XH, Lu LX, Li XZ, Jia WH (2015). Quantification of Epstein-Barr virus DNA load in nasopharyngeal brushing samples in the diagnosis of nasopharyngeal carcinoma in southern China. Cancer Sci, 106, 1196-201.

\section{(c) (i) (8)}

This work is licensed under a Creative Commons AttributionNon Commercial 4.0 International License. 